\title{
Percutaneous Disc Decompression For the Treatment of Lumbar Spinal Stenosis
}

\author{
Charles Pace, MD, PhD, Jose Reyna, MD, and Christian Schlicht, MD, PhD \\ Percutaneous disc decompression was \\ used to treat three patients with severe spinal \\ stenosis using Nucleoplasty ${ }^{\mathrm{TM}}$. Access to the \\ disc was obtained bilaterally using a parame- \\ dian approach. Channels were created bilat- \\ erally using Coblation ${ }^{\circledR}$ and stabilized using \\ coagulation. The patients were reassessed at \\ 1 week, 2 weeks, 4 weeks, 6 weeks, 6 months, \\ and 1 year. MRI was obtained on the first two \\ patients at 6 weeks. All three patients demon- \\ strated significant reduction in pain scores as \\ well as increased functionality for various pe- \\ riods of time. The use of analgesics was also \\ reduced in each of the three patients during \\ the period of diminished pain. \\ Keywords: Nucleoplasty, coblation, \\ percutaneous decompression, spinal steno- \\ sis, radiofrequency, neurogenic claudication
}

Spinal stenosis leading to neurogenic claudication is a relatively common medical condition affecting the elderly population. Typically, it is a result of several age related changes and most often secondary to a combination of a disc bulge or protrusion, bilateral facet hypertrophy, and ligamentum flavum hypertrophy (1). As spinal stenosis progresses, the patient may initially experience a variety of symptoms including muscular fatigue and/or persistent sciatic pain. Neurogenic claudication with parasthesias is characteristic of spinal stenosis but only appears in $50 \%$ of patients (2). Current medical management includes bedrest, oral non-steroidal antiinflammatory medications (NSAIDs), opioid analgesics, oral corticosteroids or epidural steroid injections (ESIs) and physical therapy. ESIs may provide temporary relief, however, efficacy often diminishes over time. Treatment with opioid analgesics may be effective, but an escalation of dosage is often necessary as the patient develops tolerance to the medication. Lumbar spinal stenosis can also be treated by open decompression surgery with or without fusion depending on the

From Division of Surgical Pain Management and Orthopedic Spine Surgery, Department of Veterans Affairs, Division of Minimally Invasive Spine Surgery, and University of New Mexico Health Center, Division of Orthopedic Surgery, Albuquerque, New Mexico. Address Correspondence: Charles Pace MD, PhD, Department of Veterans Affairs, Medical Center, Division of Minimally Invasive Spine Surgery, 1501 San Pedro Drive SE, Albuquerque, NM-87108. E-mail: cmcap928@cs.com Funding: There was no outside financial support for this project. underlying pathology. Although surgical versus medical management is controversial, numerous studies suggest that patient satisfaction is greater and functionality is improved with surgical decompression as compared to medical management (3-6). There is little difference in sustained improvement in pain scores after surgical decompression vs. medical management; however, patients often do not experience improvement in the ability to ambulate with medical management (4-6). Although surgical decompression appears to offer advantages over medical management, it requires a major operation under general anesthesia with the attendant risks of both procedures. Often, patients presenting for surgical decompression are elderly and have a variety of medical conditions. In some cases, the surgeon is unable to offer a surgical decompression because the patient is considered to be a poor surgical risk secondary to significant medical problems.

Percutaneous disc decompression has been performed for many years using a variety of techniques (7) including chemonucleolysis (8-9), automated percutaneous lumbar discectomy, percutaneous laser disc decompression (10-12), nucleoplasty (13-14), and recently via the use of the Dekompressor ${ }^{\mathrm{TM}}$ (15). Although nucleoplasty has been used for decompression of contained disk protrusions or herniations, there are no previous reports of its use for the treatment of lumbar spinal stenosis. Choy et al have proposed that the mechanism of action for laser decompression is a reduction of volume within an enclosed hydraulic space, i.e., in an intact intervertebral disc, which leads to a large change in intradiscal pressure. This results in decreased annular wall stress allowing for the annulus to retract thereby relieving compression of posterior neural elements (16). The advantages of percutaneous disc decompression vs. open procedures may be numerous, and include reduction in cost by performing an outpatient procedure, decreased mortality/morbidity from general anesthesia, a shorter recovery interval, decreased formation of scar tissue and lower incidence of nerve root injury. Moreover, there should be no need to fuse the spine secondary to instability resulting from occasional extensive laminectomies.

Recently, we have performed percutaneous disc decompression using Coblation $^{\circledR}$ (i.e., Nucleoplasty) in the treatment of severe spinal stenosis. This is a technique in which material from the disc nucleus is vaporized into elementary particles, and has been described elsewhere (13).

\section{Case Reports}

\section{Case \#1}

The first patient described is a $67-$ year-old male with severe spinal stenosis at the L4-5 level secondary to a moderate disc protrusion and severe bilateral facet hypertrophy. Initially, the patient was treated with a series of lumbar epidural steroid injections. The first injection provided excellent pain relief for nearly 4 months, whereas the second injection re- 
sulted in diminished pain for only several weeks. The patient reported symptoms of neurogenic claudication including severe sciatic pain and muscle fatigue after walking less than 1 block. Often, the patient was unable to walk the short distance to his mailbox without having to stop and rest for several minutes prior to continuing. His medical history is significant only for atypical chest pain and well-controlled hypothyroidism.

\section{Case \#2}

The second patient is a 76-yearold male with severe spinal stenosis on MRI at both the L3-4 and L4-5 levels. The pathology of his spinal stenosis included moderate disc protrusions at both levels coupled with severe bilateral facet hypertrophy and ligamentum flavum hypertrophy. His symptoms also included neurogenic claudication with severe pain, which increased throughout the day. His ability to ambulate became progressively impaired over the course of daily activities. Although he attempted to exercise using a treadmill, he could walk only 10-15 minutes using the slowest speed setting and no incline. When this patient was evaluated after referral from an outside facility, he was taking 500mg naproxyn bid, and ibuprofen $600 \mathrm{mg}$ bid. This dangerous combination provided only mild relief.

\section{Case \#3}

The third patient is a 53-year-old male also with severe spinal stenosis at the L3-4 and L4-5 levels due to congenitally short pedicles and large bulging discs at both levels. Previously, he had a positive provocative response to discography at L4-5 and L5-S1, and an annular disc tear at L5-S1, which had been treated with intradiscal electrothermal annuloplasty. His back pain was treated successfully with this modality; however, he continued to have symptoms of neurogenic claudication. His stenosis was most severe at the L4-5 level, and prior to treatment, he was limited to ambulating only 1 block or standing several minutes prior to the onset of severe lower extremity pain causing him to sit and rest before continuing. He also reported increasing pain and muscle fatigue during his activities of daily living. This patient reported taking Darvocet ${ }^{\circledR}$ 4-6 tabs/day at minimum prior to treatment.

Each of the three patients described above was considered a surgical candidate, however, each was referred for percutaneous decompression secondary to their strong preference to seek a less invasive method of treatment.

\section{Methods}

Each of the three patients described was treated on an outpatient basis. After obtaining informed consent, each pa- tient was moved to the operating room, and placed on the table in the prone position. The procedure was performed in a sterile manner; under fluoroscopic guidance, two 17ga Crawford needles were advanced into the lumbar disc of interest bilaterally using a paramedian approach. Proper placement was confirmed with AP and lateral fluoroscopic views (Fig. 1). Radio opaque contrast $1.5 \mathrm{~mL}$ with $200 \mathrm{mg} / \mathrm{mL}$ cefazolin was injected into the disc nucleus. The Perc-D SpineWand ${ }^{\mathrm{TM}}$ was advanced through the Crawford needle and the anterior and posterior margins of the nucleus were defined. Then, using power level 2, channels were created and stabilized as described elsewhere (13-14). The procedure was then repeated by inserting the SpineWand into the opposite introducer needle and creating additional channels. In the case of multilevel disease, the procedure of obtaining disc access and performing Nucleoplasty were repeated at the second disc level. In all three cases, the procedure was done with local anesthetic, and with or without mild conscious IV sedation in addition to local anesthesia. Post procedure, the patients were discharged home after approximately 90 minutes recovery with instructions to wear an abdominal binder during awake hours for a period of 10 days, and to limit lifting to less than 20 pounds for 4 weeks.

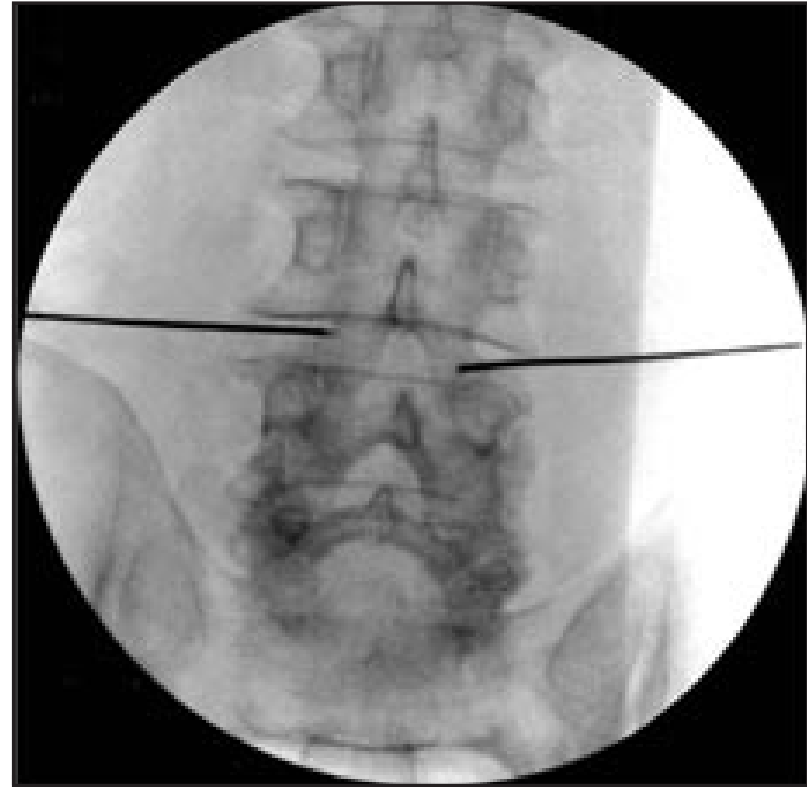

Fig 1. 17ga introducer needles placed into the L3-4 disc bilaterally.

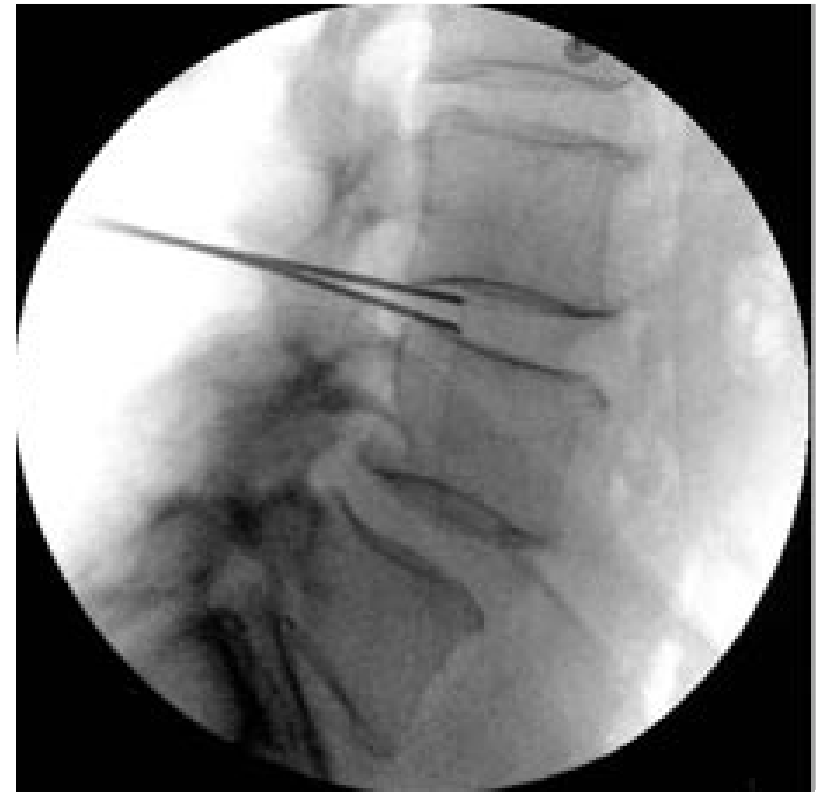

Fig 1A. Anterior-posterior view 


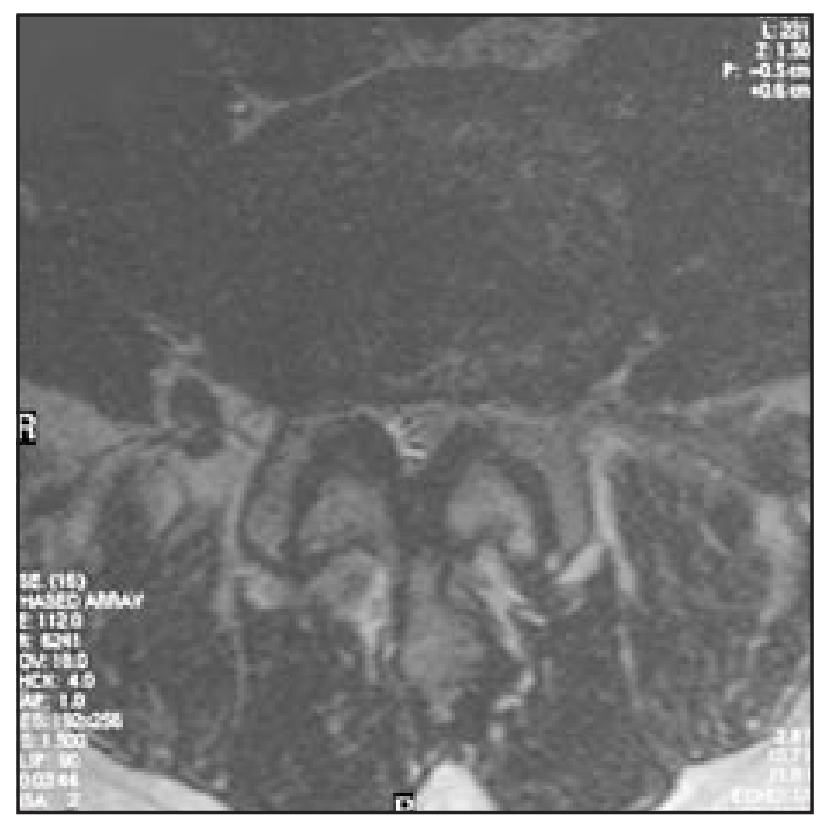

Fig 2A. MRI image before Nucleoplasty

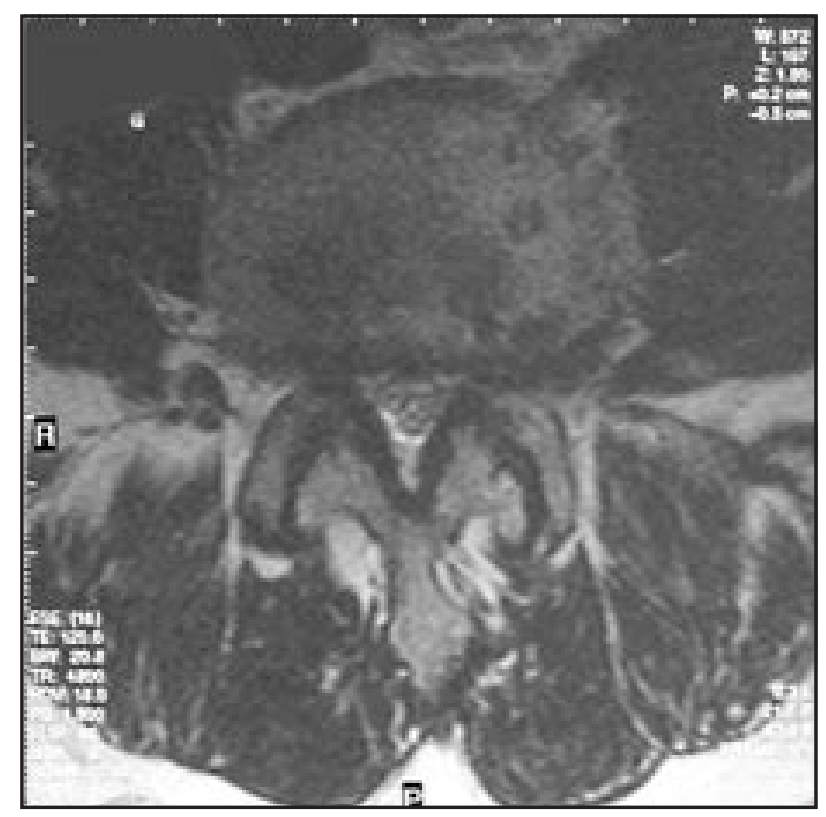

Fig 2B. MRI image 6 weeks post nucleoplasty

\section{RESULTS}

The first patient had the Nucleoplasty procedure performed at the L4-5 level using a bilateral approach and creating 6 channels in the nucleus pulposis (NP) bilaterally. In theory, this process should create approximately $3 \mathrm{~mL}$ of volume into which the disc bulge may retract. However, in elderly patients with relatively dehydrated discs, it is likely that less volume is actually created. The second patient was treated with Nucleoplasty at both the L3-4 and L4-5 levels again using a bilateral approach at each level, and creating 6 channels in the NP bilaterally at L3-4, and 8 channels in the NP bilaterally at the L4-5 level. The pre-procedure MRI of the L45 level demonstrated near obliteration of the canal by the disc protrusion and facet hypertrophy. The third patient was treated similarly using decompression Nucleoplasty at the L3-4 and L4-5 levels. Again, each level was treated bilaterally creating 6 channels per side at L3-4, and 8 channels per side at L4-5.

Each patient was followed at 1 week, 2 weeks, 4 weeks, and 6 weeks, 6 months, and one year. Each reported significant improvement in functionality as well as reduction in pain levels for various intervals. The first patient reported significant relief after 1 week, which continued for 9 months at which time he began to note a recurrence of symptoms. One-year post procedure, the patient reported worsen- ing symptoms of neurogenic claudication, however, his pain and diminished functionality were still only $50 \%$ of baseline prior to undergoing the percutaneous disc decompression. He has elected to repeat the procedure in the near future as opposed to undergoing a surgical evaluation. During the interval of improvement he reported walking $>1 / 4$ mile numerous times per day without the return of symptoms as well as being able to participate in activities of daily living with little limitation. He reported needing analgesics (ibuprofen) only 2-3 times per week during that interval. MRI repeated 6 weeks post-decompression showed a definite decrease in the size of the disc bulge on axial images (Fig. 2).

The second patient reported that 1 week post-procedure, he was able to walk on a treadmill for up to 40 minutes at a speed setting of 3 with a slight incline. Subjectively, his pain was reduced by greater than $70 \%$ for a period of $4-5$ months post-decompression after which time his symptoms again began to worsen. During that interval, he reduced his daily analgesics to $200 \mathrm{mg}$ etodolac/day. Repeat MRI demonstrate only slight improvement on the axial images at both levels. Eight months post-procedure, the patient reported his pain and functionality had returned to baseline. He opted for a surgical evaluation and subsequently underwent a 2 level open decompression 2 months later.
The third patient also reported significant improvement of symptoms beginning on the first postoperative day, and lasting 11 months prior to his beginning to note a recurrence of symptoms. During the interval of improvement, however, he had increased his ambulatory capacity to $>1$ mile with no symptoms and his analgesic requirement decreased to 12 tabs of to propoxyphene 1-2 days/week. His repeat MRI 6 weeks post-decompression also demonstrated an increase in the size of the central canal compared to his previous film.

\section{DISCUSSION}

Lumbar spinal stenosis is a significant medical issue for elderly patients. Although medical treatment options are available, they do not significantly improve functionality even though pain scores are similar to post surgical patients. The process of correcting the abnormal anatomy to widen the central canal (i.e., discectomy with or without fusion) necessarily disturbs the normal anatomy by the very nature of the surgical intervention. This may lead to formation of adhesions, which can result in compression of neural elements leading to chronic pain. In addition, lumbar fusion may be necessary to correct instability resulting from extensive laminectomies in open surgical cases. Herein, we propose that percutaneous disc decompression may provide at least a temporary improvement 
in pain scores and increased functionality in properly selected patients with lumbar spinal stenosis when a disc bulge provides a significant contribution to the pathology. All three of the patients treated in this manner reported increased ambulatory capacity, decreased pain levels, and decreased usage of analgesics for a period ranging from 4-10 months. By creating additional volume within the nucleus pulposis, inward disc retraction with decreased compression of posterior neural elements is the most likely mechanism as evidenced by improvement in symptoms as well as MRI imaging. The procedure is easily done on an outpatient basis, and does not compromise the surgeons' ability to perform an open procedure in the future should such a procedure become necessary. The sample size used in this small study is an obvious limitation, and extrapolation to a larger population may not be possible, however, we note that all three patients reported significant improvement, and in one case, the patient was markedly improved the following day. Although each of the three patients were acceptable surgical candidates, they all preferred a minimally invasive procedure to an open operation, and only one of the three patients opted for open surgical decompression when symptoms recurred. The procedure can easily be accomplished with local anesthesia and mild IV sedation, and can be done on patients with significant underlying medical problems for whom an open operation may not be considered due to the risk of a general anesthetic. Of note, we feel that not all patients with spinal stenosis will be candidates for this procedure, or other percutaneous decompression techniques. Unless there is a disc bulge that contributes significantly to the pathology, the procedure is unlikely to result in improvement. Moreover, in elderly patients with significant degenerative disc disease and poorly hydrated discs, it is unclear as to whether an adequate amount of NP will be removed, and thus, there may be no change in the morphology of the disc. Nevertheless, a significant population of patients may benefit from percutaneous disc decompression in this manner. Although we used nucleoplasty as our disc decompression procedure, any percutaneous method, such as LASETM or the Dekompressor $^{\mathrm{TM}}$ method may be as likely to achieve success. Unfortunately, with the possible exception of the Dekompressor method, it is difficult to quantify the amount of material removed. Although MRI may be helpful in this regard, a decompression procedure in which the extent of disc material removed could be easily quantified would be desirable. Obviously, this technique should be evaluated using validated assessment tools, an adequate sample size, and a control group with patients randomized to medical management vs. percutaneous decompression. Patients with severely dehydrated discs, as seen on MRI are unlikely to benefit significantly from this procedure, and should probably be excluded from a formalized study. Moreover, the annulus in these patients may be so calcified that no change in disc morphology may occur in any case. Thus, a formalized study using accepted assessment tools to evaluate patient improvement along with pre and post MRI results would aid in determining the efficacy of this technique compared to non-invasive treatment modalities.

\section{CONCLUSION}

Although this is a small case study with limitations as noted, we believe that percutaneous disc decompression may be a viable option in carefully selected patients. It may be especially beneficial in patients with severe spinal stenosis who are not candidates for open decompression due to other medical conditions. Our observations suggest that increasing the diameter of the central canal by only a small amount can provide significant pain relief. It is likely that patients with preserved disc height and well-hydrated discs may obtain significant relief of the symptoms of neurogenic claudication, whereas patients with severely dehydrated discs may not benefit from the procedure. Clearly, a well-controlled trial will help to determine whether this treatment modality can be used to provide cost-effective care to patients with severe spinal stenosis.

\section{RefERENCES}

1. Finch PM, Taylor JR. Functional anatomy of the spine. In Waldman SD (ed). Interventional Pain Management. W.B. Saunders Company, Philadelphia, 2001, pp 43-71.

2. Maigne R. Clinical Forms of Sciatica. In Pain of Vertebral Origin. Williams and Wilkins, Baltimore, 1996, pp 330-334.

3. Simotas AC, Dorey FJ, Hansraj KK et al. Nonoperative treatment for lumbar spinal stenosis. Spine 2000; 25:197-204.

4. Taylor VM, Deyo RA, Ciol M et al. Patient-

\section{Author Affiliation:}

Charles Pace, MD, PhD

Department of Veterans Affairs

Medical Center

Division of Minimally Invasive Spine

Surgery

1501 San Pedro Drive SE

Albuquerque, New Mexico-87108

\section{Jose Reyna, MD}

University of New Mexico Health

Center

Division of Orthopedic Surgery

Albuquerque, New Mexico-87120

\section{Christian Schlicht, MD, PhD}

Department of Veterans Affairs

Medical Center

Division of Minimally Invasive Spine

surgery

1501 San Pedro Drive SE

Albuquerque, New Mexico-87108

oriented outcomes from low back surgery. Spine 2000; 25:2445-2452.

5. Atlas SJ, Keller RB, Robson BS, et al. Surgical and nonsurgical management of lumbar spinal stenosis. Spine 2000; 25:556562.

6. Amundsen $\mathrm{T}$, Weber $\mathrm{H}$, Nordal $\mathrm{HJ}$ et al. Lumbar spinal stenosis: conservative or surgical management? Spine 2000; 25: 1424-1436.

7. Mayer, HM. Percutaneous lumbar disc surgery. Spine 1994; 19:2719-2723.

8. Smith LW. Enzyme dissolution on nucleus pulposus in humans. Clin Ortho Related Res 1986; 206:4-9.

9. Brown MD. Update on chemonucleolysis. Spine 1996; 21:62S-68S.

10. Choy DS, Ascher PW, Ranu HS et al. Percutaneous laser disc decompression: A new therapeutic modality. Spine 1992; 17:949956.

11. Choy DS, Ngeow J. Percutaneous laser disc decompression in spinal stenosis. J Clin Laser Med Surg 1998; 16:123-125.

12. Choy DSJ. Percutaneous laser disc decompression (PLDD): Twelve years' experience with 752 procedures in 518 patients. J Clin Laser Med Surg 1998; 16:325-331.

13. Sharps LS, Isaac Z. Percutaneous disc decompression using nucleoplasty. Pain Physician 2002; 5:121-126.

14. Singh V, Piryani C, Liao K et al. Percutaneous disc decompression using coblation (nucleoplasty) in the treatment of chronic discogenic pain. Pain Physician 2002; 5: 250-259.

15. Vilims BD, Brandt SA, Wright RE. Percutaneous disc decompression/ diskectomy. Prac tical Pain Management. 2002; 2:19-22.

16. Choy DSJ, Altman P. Fall of intradiscal pressure with laser ablation. Spine 1993; 7:23-29. 